\title{
ON FUCHSIAN GROUPS OF ACCESSIBLE TYPE
}

\author{
CH. POMMERENKE
}

\section{Introduction}

1.1. Let $\Gamma$ be a Fuchsian group acting on the open unit disk $\boldsymbol{D}$. The group $\Gamma$ is called of accessible type if there exists a measurable set $B \subset \partial \boldsymbol{D}$ of positive Lebesgue measure that contains no two $\Gamma$-equivalent points, and $\Gamma$ is called of fully accessible type if moreover

$$
\partial \boldsymbol{D} \doteq \Gamma B \equiv \bigcup_{\gamma \in \boldsymbol{\Gamma}} \gamma(B)
$$

where $\stackrel{\circ}{=}$ denotes equality up to a set of zero measure. Every group of accessible type is of convergence type and therefore has a Green's function.

We define

$$
u(\zeta)=\sum_{\gamma \in \Gamma}\left|\gamma^{\prime}(\zeta)\right| \text { for } \zeta \in \partial D .
$$

It was shown in [10] and [11] that the following conditions are equivalent:

(i) $\Gamma$ is of fully accessible type;

(ii) for almost all $\zeta \in \partial D$, every horocycle at $\zeta$ contains only finitely many points $\gamma(0)$ with $\gamma \in \Gamma$

(iii) $u(\zeta)<\infty$ for almost all $\zeta \in \partial D$;

(iv) the Green's measure of $\Gamma$ is absolutely continuous with density $u$. The Green's measure of $\Gamma$ is the reformulation [10] for the groups $\Gamma$ of the Green's measure (Brelot and Choquet [1]) of the Riemann surface $\boldsymbol{D} / \Gamma$.

D. Sullivan [16, Theorem III] has shown that (i) is also equivalent to the condition

(v) for almost all $\zeta \in \partial \boldsymbol{D}$, some horocycle at $\zeta$ contains no $\gamma(0)(\gamma \in \Gamma)$.

He considers the ergodic properties of the action of $\Gamma$ on $\partial \boldsymbol{D}$ and actually generalizes (i) $\Leftrightarrow($ ii) $\Leftrightarrow($ iii) to Kleinian groups of any dimension. In the present case we have

$$
\text { (dissipative part of } \partial \boldsymbol{D}) \stackrel{\circ}{=} \bigcup_{\gamma \in \Gamma} \gamma(\partial \boldsymbol{D} \cap \partial F)
$$

where $F$ denotes a Ford fundamental domain of $\Gamma$. Thus "not accessible" means that $\partial \boldsymbol{D}$ is conservative while "fully accessible" means that $\partial \boldsymbol{D}$ is dissipative with respect to the action of $\Gamma$ on $\partial \boldsymbol{D}$. 
S. J. Patterson has proved [7, Theorem 3] that $\Gamma$ is of fully accessible type if the exponent of convergence satisfies $\delta(\Gamma)<1 / 2$, that is if $\sum_{\gamma \in \Gamma}\left|\gamma^{\prime}(0)\right|^{\alpha}<\infty$ for some $\alpha<1 / 2$. On the other hand, he has constructed [8] an example of a group with $\delta(\Gamma)<1$ which is not even of accessible type.

1.2. As a motivation we briefly consider the analogue of the Martin boundary for the group $\Gamma$. The Martin boundary is discussed in the book of Constantinescu and Cornea [2] for hyperbolic Riemann surfaces.

Let $\Gamma$ be of fully accessible type. It follows from (1.2) and (iii) that, for almost all $\zeta \in \partial D$, the "Poisson kernel"

$$
p(z, \zeta)=\frac{1}{u(\zeta)} \sum_{\gamma \in \Gamma} \frac{1-|\gamma(z)|^{2}}{|\zeta-\gamma(z)|^{2}}=\sum_{\gamma \in \Gamma} \frac{1-|z|^{2}}{|\gamma(\zeta)-z|^{2}} \frac{\left|\gamma^{\prime}(\zeta)\right|}{u(\zeta)}
$$

is a positive harmonic function of $z \in \boldsymbol{D}$ with $p(0, \zeta)=1$ and $p(\gamma(z), \zeta)=p(z, \zeta)$ for $\gamma \in \Gamma$. It is minimal among functions with these properties. If $v$ is a bounded harmonic function in $\boldsymbol{D}$ with $v \circ \gamma=v$ for $\gamma \in \Gamma$ then, by the Poisson integral formula and by (1.1) and (1.3),

$$
\begin{gathered}
v(z)=\frac{1}{2 \pi} \int_{\partial D} \frac{1-|z|^{2}}{|\zeta-z|^{2}} v(\zeta)|d \zeta|=\sum_{\gamma \in \Gamma} \frac{1}{2 \pi} \int_{\gamma(B)} \frac{1-|z|^{2}}{|\zeta-z|^{2}} v(\zeta)|d \zeta| \\
=\frac{1}{2 \pi} \int_{B} p(z, \zeta) v(\zeta) u(\zeta)|d \zeta|
\end{gathered}
$$

and conversely the last integral always represents a $\Gamma$-invariant bounded harmonic function. It follows [2, p. 138] that almost all points of the Martin boundary of $\boldsymbol{D} / \Gamma$ can be represented by points of $B$; the canonical measure becomes $u(\zeta)|d \zeta|$. In particular, if $A$ is a $\Gamma$-invariant measurable set on $\partial D$ then, by (1.4),

$$
\omega(z, A, \boldsymbol{D})=\frac{1}{2 \pi} \int_{\boldsymbol{B} \cap \boldsymbol{A}} p(z, \zeta)|u(\zeta)||d \zeta| \quad(z \in \boldsymbol{D})
$$

is the harmonic measure of $A$ with respect to $D$.

I want to thank the referee for his helpful comments. The present form of Theorem 1 and its proof is due to him.

\section{Characterizations in the disk}

2.1. Let $\Gamma$ be a Fuchsian group with identity $\imath$ and limit set $L(\Gamma)$. The Ford fundamental domain is

$$
F=\left\{z \in \boldsymbol{D}:\left|\gamma^{\prime}(z)\right|<1 \text { for } \gamma \in \Gamma, \gamma \neq i\right\} .
$$

Let mes denote the Lebesgue measure on $\partial \boldsymbol{D}$. 
The group $\Gamma$ is [10] of accessible type if and only if mes $(\partial F \cap \partial \boldsymbol{D})>0$, and it is of fully accessible type if and only if

$$
\partial \boldsymbol{D} \doteq \Gamma(\partial F \cap \partial D)=\bigcup_{\gamma \in \Gamma} \gamma(\partial F \cap \partial D) ;
$$

this is a disjoint union except for countably many points. Since always

$$
\partial \boldsymbol{D}=L(\Gamma) \cup \Gamma(\partial F \cap \partial \boldsymbol{D})
$$

we see that all groups with mes $L(\Gamma)=0$ are of fully accessible type. This holds in particular for finitely generated groups of the second kind.

Theorem 1. Let there exist measurable sets $A_{n} \subset \partial D$ with

$$
\begin{array}{ccc}
\operatorname{mes} A_{n} \rightarrow 2 \pi & \text { as } & n \rightarrow \infty, \\
\gamma\left(A_{n}\right) \cap A_{n}=\emptyset & \text { for } & \gamma \in \Gamma \backslash \Gamma_{n}
\end{array}
$$

for $n=1,2, \ldots$ where either

(a) $\Gamma_{n}$ is a finite set, or

(b) $\Gamma_{n}$ is a subgroup of $\Gamma$ of fully accessible type, or

(c) $\Gamma$ is infinitely generated and $\Gamma_{n}$ is a finitely generated subgroup.

Then $\Gamma$ is of fully accessible type.

Proof. We prove first that there exist sets $C_{n} \subset A_{n}(n=1,2, \ldots)$ such that

$$
\Gamma A_{n}=\Gamma C_{n}, \quad \gamma\left(C_{n}\right) \cap C_{n}=\emptyset \text { for } \gamma \in \Gamma \backslash\{l\} .
$$

In case (a) the set $A_{n} \cap\{\gamma(\zeta): \gamma \in \Gamma\}$ is finite for each $\zeta \in A_{n}$ and therefore has a point with smallest argument $\in[0,2 \pi]$. These points together form a measurable set $C_{n}$ satisfying (2.6).

Let now (b) hold. Then $\Gamma_{n} A_{n}$ satisfies (2.4) and also (2.5) because $\Gamma_{n}$ is a group. Hence we may assume that $A_{n}$ is $\Gamma_{n}$-invariant. Since $\Gamma_{n}$ is of fully accessible type there exist measurable sets $B_{n} \subset \partial D$ containing no two $\Gamma_{n}$-equivalent points such that $\partial D \doteq \Gamma_{n} B_{n}$. Hence

$$
\Gamma A_{n} \stackrel{\circ}{=} \Gamma\left(A_{n} \cap \Gamma_{n} B_{n}\right)=\Gamma\left(\Gamma_{n} A_{n} \cap \Gamma_{n} B_{n}\right)=\Gamma\left(A_{n} \cap B_{n}\right)
$$

and this proves (2.6) with $C_{n}=A_{n} \cap B_{n}$ because of (2.5).

Let finally (c) be satisfied. Since $\Gamma$ is not finitely generated $F$ has infinite noneuclidean area [3, p. 210]. Hence the Ford fundamental domain of $\Gamma_{n}$ has also infinite area. Therefore $\Gamma_{n}$ is of the second kind and thus of fully accessible type so that case (b) applies. This proves (2.6) for all three cases.

We define now

$$
E_{n}=C_{n} \backslash \bigcup_{k=1}^{n-1} \Gamma C_{k}
$$


and $B=\bigcup_{n} E_{n}$. It follows from (2.6) that $\gamma(B) \cap B=\emptyset$ for $\gamma \in \Gamma \backslash\{l\}$. Furthermore, by (2.6),

$$
\Gamma E_{n} \supset \Gamma A_{n} \backslash \bigcup_{k=1}^{n-1} \Gamma A_{k}, \text { thus } \Gamma B \supset \bigcup_{n=1}^{\infty} \Gamma A_{n},
$$

and we conclude from (2.4) that $\partial \boldsymbol{D} \doteq \Gamma B$.

We consider the symmetric Stolz angle of opening $2 \alpha$ at $\zeta \in \partial \boldsymbol{D}$, namely

$$
\Delta_{\alpha}(\zeta, \delta)=\{z \in \boldsymbol{D}:|\arg (1-\bar{\zeta} z)|<\alpha,|z-\zeta|<\delta\} \quad(0<\delta<1) .
$$

Corollary 1. Let $G_{n} \subset \boldsymbol{D}$ be open sets and let

(2.8) $A_{n}=\left\{\zeta \in \partial D: \Delta_{\alpha}(\zeta, \delta) \subset G_{n}\right.$ for some $\left.\alpha=\alpha_{n}(\zeta)>0, \quad \delta=\delta_{n}(\zeta)>0\right\}$.

If mes $A_{n} \rightarrow 2 \pi$ as $n \rightarrow \infty$ and if, for $n=1,2, \ldots$,

$$
\gamma\left(G_{n}\right) \cap G_{n}=\emptyset \text { for } \gamma \in \Gamma \backslash \Gamma_{n}
$$

where $\Gamma_{n}$ satisfies one of the conditions (a)-(c) of Theorem 1. Then $\Gamma$ is of fully accessible type.

Proof. If $\gamma\left(A_{n}\right) \cap A_{n} \neq \emptyset$ it follows from (2.8) by a simple geometric argument that $\gamma\left(G_{n}\right) \cap G_{n} \neq \emptyset$. Hence (2.9) implies (2.5), and $\Gamma$ is of fully accessible type by Theorem 1 .

2.2. We give now a characterization in terms of the Ford fundamental domain $F$. Let $l$ denote the length.

Theorem 2. The group $\Gamma$ is of fully accessible type if and only if there are domains $G_{n} \subset \boldsymbol{D}$ bounded by rectifiable Jordan curves such that

$$
G_{n} \rightarrow D, \quad l\left(D \cap \partial G_{n}\right) \rightarrow 0 \quad \text { as } n \rightarrow \infty
$$

and, for some finite set $\Gamma_{n} \subset \Gamma$,

$$
\gamma(\bar{F}) \cap G_{n}=\emptyset \quad \text { for } \quad \gamma \in \Gamma \backslash \Gamma_{n} .
$$

We can express this also by saying that, for each $\varepsilon>0$, there exist disks $D_{k}$ such that

$$
\bigcup_{\gamma \in I \backslash I_{n}} \gamma(\bar{F}) \subset \bigcup_{k} D_{k}, \sum_{k} \operatorname{diam} D_{k}<\varepsilon ;
$$

note that, for general Fuchsian groups, this relation only holds with (diam $\left.D_{k}\right)^{2}$ instead of diam $D_{k}$.

Proof. (a) Let $\Gamma=\left\{\gamma_{v}: v=1,2, \ldots\right\}$ be of fully accessible type and let

$$
H_{n}=\bigcup_{v=1}^{n} \gamma_{v}(F) \cup(\text { intermediate sides }) \quad(n=1,2, \ldots) .
$$


Since the curve $\partial F$ is rectifiable it has a tangent almost everywhere. Hence, by (2.2), there are sets $A_{n} \subset \partial D(n=1,2, \ldots)$ with

$$
\operatorname{mes} A_{n}=\sum_{\nu=1}^{n} \operatorname{mes}\left(\partial \gamma_{v}(F) \cap \partial D\right) \rightarrow 2 \pi \quad(n \rightarrow \infty)
$$

such that $\Delta\left(\zeta, \delta_{n}(\zeta)\right) \subset H_{n}$ for every $\zeta \in A_{n}$ and some $\delta_{n}(\zeta)>0$ where $\Delta \equiv \Delta_{\pi / 4}$ as in (2.7). Since

$$
A_{n}=\bigcup_{k=1}^{\infty}\left\{\zeta \in A_{n}: \Delta(\zeta, 1 / k) \subset H_{n}\right\} \quad(n=1,2, \ldots)
$$

we can find $k_{n}$ such that

$$
B_{n} \equiv\left\{\zeta \in A_{n}: \Delta\left(\zeta, 1 / k_{n}\right) \subset H_{n}\right\} \text {, mes } B_{n} \rightarrow 2 \pi \quad(n \rightarrow \infty) .
$$

Let $D_{n}=\left\{|z|<1-1 /\left(5 k_{n}\right)\right\}$. Then

$$
G_{n}=D_{n} \cup \bigcup_{\zeta \in B_{n}} \Delta\left(\zeta, 1 / k_{n}\right)
$$

is a starlike domain with $B_{n} \subset \partial G_{n}$ and, under the projection mapping

$$
z \in \partial G_{n} \mapsto \frac{z}{|z|} \in \partial D,
$$

lengths are decreased at most by a factor $1 / 1 / 2$. Hence

$$
l\left(\boldsymbol{D} \cap \partial G_{n}\right) \leqq \sqrt{2}\left(2 \pi-\operatorname{mes} B_{n}\right) \rightarrow 0 \quad(n \rightarrow \infty)
$$

because of (2.15). Since $\Gamma$ is discontinuous there exist $N_{n} \geqq n$ such that $D_{n} \subset H_{N_{n}}$. Then it follows from (2.15) and (2.16) that $G_{n} \subset H_{N_{n}}$. Hence we conclude from (2.13) that

$$
\gamma_{v}(\bar{F}) \cap G_{n} \subset \gamma_{v}(\bar{F}) \cap H_{N_{n}}=\emptyset \quad \text { for } \quad v>N_{n} .
$$

Thus (2.9) holds with $\Gamma_{n}=\left\{\gamma_{v}: v=1, \ldots, N_{n}\right\}$.

(b) Conversely let there exist domains $G_{n}$ with (2.10) and (2.11). Since the rectifiable curve $\partial G_{n}$ has a tangent almost everywhere and since $l\left(\partial D \cap \partial G_{n}\right) \rightarrow 2 \pi(n \rightarrow \infty)$ by (2.10), the sets $A_{n}$ defined by (2.8) satisfy mes $A_{n} \rightarrow 2 \pi$ as $n \rightarrow \infty$. If $\Gamma_{n=}^{*}=$ $\left\{\alpha \circ \beta^{-1}: \alpha, \beta \in \Gamma_{n}\right\}$ then $\gamma\left(G_{n}\right) \cap G_{n}=\emptyset$ for $\gamma \in \Gamma \backslash \Gamma_{n}^{*}$. Since $\Gamma$ is finite it therefore follo:ys from Corollary 1 that $\Gamma$ is of fully accessible type.

\section{Characterization as a Riemann surface}

Let $\cong$ denote conformal equivalence. The Riemann surface $R \cong \boldsymbol{D} / \Gamma$ is said to be of finite topological type if it has finite genus and finite connectivity. This holds if and only if the Fuchsian group $\Gamma$ is finitely generated; see for instance [3, p. 200]. If $R$ is of finite topological type and not a punctured compact surface then $\Gamma$ is also 
of the second kind and thus of fully accessible type; see Section 2 . We will then denote by $\omega(p, E, R)$ the harmonic measure of the boundary set $E$ at the point $p \in R$.

Theorem 3. Let $R \cong \boldsymbol{D} / \Gamma$ be a Riemann surface of infinite topological type and let $p \in R$. Then $\Gamma$ is of fully accessible type if and only if there exist surfaces $R_{n}$ of finite topological type with $p \in R_{n} \subset R$ such that

$$
\omega\left(p, \partial R \cap \partial R_{n}, R_{n}\right) \rightarrow 1 \text { as } n \rightarrow \infty .
$$

Since $R_{n}$ is a compact bordered surface (possibly with finitely many punctures) the set $\partial R \cap \partial R_{n}$ lies on this border and we do not have to consider ideal boundaries.

Proof. (a) Let there first exist subsurfaces $R_{n}$ of finite topological type such that (3.1) holds. We may assume that $R \backslash R_{n}$ has no relatively compact components because "filling in the holes" increases the harmonic measure. We may furthermore assume that $R=\boldsymbol{D} / \Gamma$ and $\pi(0)=p$ where $\pi$ denotes the projection map of $\boldsymbol{D}$ onto $\boldsymbol{D} / \Gamma$.

Let $G_{n}$ be the component of $\pi^{-1}\left(R_{n}\right) \subset \boldsymbol{D}$ that contains 0 and let $\Gamma_{n}=\{\gamma \in \Gamma$ : $\left.\gamma\left(G_{n}\right)=G_{n}\right\}$. Then $G_{n} \cap \gamma\left(G_{n}\right)=\emptyset$ for $\gamma \in \Gamma \backslash \Gamma_{n}$. Furthermore $G_{n}$ is simply connected in $D$ because $R \backslash R_{n}$ does not possess a relatively compact component. Let $f_{n}$ map $D$ conformally onto $G_{n}$ such that $f_{n}(0)=0$. Then

$$
\Gamma_{n}^{*}=\left\{f_{n}^{-1} \circ \gamma \circ f_{n}: \gamma \in \Gamma_{n}\right\}
$$

is a Fuchsian group in $D$ with $R_{n} \cong G_{n} / \Gamma_{n} \cong \boldsymbol{D} / \Gamma_{n}^{*}$. Since $R_{n}$ is of finite topological type the group $\Gamma_{n}^{*}$ is finitely generated hence also $\Gamma_{n}$. Since $\Gamma$ is infinitely generated $\Gamma_{n}$ is of the second kind.

The projection map of $D$ onto $R_{n} \cong \boldsymbol{D} / \Gamma_{n}^{*}$ is $\pi \circ f_{n}$. If we define $C_{n}=$ $f_{n}^{-1} \circ \pi^{-1}\left(\partial R \cap \partial R_{n}\right)$ then

$$
\omega\left(\pi \circ f_{n}(z), \partial R \cap \partial R_{n}, R_{n}\right)=\omega\left(z, C_{n}, \boldsymbol{D}\right) \quad(z \in \boldsymbol{D}) .
$$

Since $f_{n}(0)=0$ and $\pi(0)=p$ it follows from (3.1) that

$$
\text { mes } C_{n}=2 \pi \omega\left(0, C_{n}, D\right) \rightarrow 2 \pi \quad \text { as } n \rightarrow \infty .
$$

The set $f_{n}\left(C_{n}\right)$ lies on $\partial \boldsymbol{D}$. Hence we conclude from a generalisation of Löwner's lemma [4, Lemma 1], [6, p. 57] that mes $f_{n}\left(C_{n}\right) \geqq \operatorname{mes} C_{n} \rightarrow 2 \pi$ as $n \rightarrow \infty$. By the McMillan twist point theorem [5], [9, p. 326] the function $f_{n}$ has a finite angular derivative at almost all points of $C_{n}$. It follows [9, p. 303] that (2.10) holds for some set $A_{n}$ with mes $A_{n}=$ mes $f\left(C_{n}\right)$. Hence mes $A_{n} \rightarrow 2 \pi$ as $n \rightarrow \infty$, and $\Gamma$ is therefore of fully accessible type by the corollary.

We need the following known lemma to prove the converse.

Lemma. Let $G_{n}$ be Jordan domains with $0 \in G_{n}$ and let $f_{n}$ map $\boldsymbol{D}$ conformally onto $G_{n}$ such that $f_{n}(0)=0$. If

$$
l\left(\partial G_{n}\right) \rightarrow 2 \pi, \quad\left|f_{n}^{\prime}(0)\right| \rightarrow 1 \quad \text { as } \quad n \rightarrow \infty
$$


then, for all measurable sets $C_{n} \subset \partial D$,

$$
l\left(f\left(C_{n}\right)\right)-\operatorname{mes} C_{n} \rightarrow 0 \text { as } n \rightarrow \infty \text {. }
$$

Proof. Let $a_{n}=\sqrt{f_{n}^{\prime}(0)}$; note that $\left|a_{n}\right| \rightarrow 1$ by (3.2). We write

$$
\sqrt{f_{n}^{\prime}(z)}=a_{n}+g_{n}(z)(z \in D), g_{n}(0)=0 \text {. }
$$

Then it follows from Parseval's formula and from (3.2) that

$$
\frac{1}{2 \pi} \int_{\partial D}\left|g_{n}(z)\right|^{2}|d z|=\frac{1}{2 \pi} \int_{\partial D}\left|f_{n}^{\prime}(z)\right||d z|-\left|a_{n}\right|^{2}=l\left(\partial G_{n}\right) / 2 \pi-\left|a_{n}\right|^{2} \rightarrow 0
$$

as $n \rightarrow \infty$. Furthermore, by (3.4),

$$
l\left(f\left(C_{n}\right)\right)-\left|a_{n}\right|^{2} \text { mes } C_{n}=\int_{C_{n}}\left(2 \operatorname{Re}\left[\bar{a}_{n} g_{n}(z)\right]+\left|g_{n}(z)\right|^{2}\right)|d z|
$$

Hence (3.3) follows from (3.5) by the Schwarz inequality.

Proof of Theorem 3. (b) Let now $\Gamma$ be of fully accessible type. Let $G_{n}$ be the Jordan domains defined by (2.8) in the proof of Theorem 1 . Since $\left(G_{n}\right)$ is an exhaustion of $\boldsymbol{D}$ and since $l\left(\boldsymbol{D} \cap \partial G_{n}\right) \rightarrow 0$ as $n \rightarrow \infty$, we see that (3.2) holds. We can write $\partial \boldsymbol{D} \cap \partial G_{n}=f_{n}\left(C_{n}\right)$ with $C_{n} \subset \partial \boldsymbol{D}$. Since $l\left(f_{n}\left(C_{n}\right)\right)=l\left(\partial G_{n}\right)-l\left(\boldsymbol{D} \cap \partial G_{n}\right) \rightarrow 2 \pi \quad$ as $n \rightarrow \infty$ by (2.3), it follows from the lemma that

$$
\omega\left(0, \partial D \cap \partial G_{n}, G_{n}\right)=\omega\left(0, C_{n}, \boldsymbol{D}\right)=\frac{\operatorname{mes} C_{n}}{2 \pi} \rightarrow 1(n \rightarrow \infty) .
$$

Let $H_{n}$ be the open set defined by (2.5). Since $G_{n} \subset H_{n}^{*} \equiv H_{N_{n}}$ it follows from the principle of domain extension [6, p. 69] and from (3.6) that

$$
\omega\left(0, \partial \boldsymbol{D} \cap \partial H_{n}^{*}, H_{n}^{*}\right) \geqq \omega\left(0, \partial \boldsymbol{D} \cap \partial G_{n}, H_{n}^{*}\right) \geqq \omega\left(0, \partial \boldsymbol{D} \cap \partial G_{n}, G_{n}\right) \rightarrow 1
$$

as $n \rightarrow \infty$. We define $R_{n}=\pi\left(H_{n}^{*}\right)$, the projection of $H_{n}^{*}$ into $R$. Since $\omega\left(p, \partial R \cap \partial R_{n}, R_{n}\right)=\omega\left(0, \partial \boldsymbol{D} \cap \partial H_{n}^{*}, H_{n}^{*}\right)$ the assertion (3.1) follows from (3.7).

We see from (2.5) that

$$
H_{n}^{*}=\bigcup_{v=1}^{N_{n}} \gamma_{v}(F) \cup(\text { intermediate sides })
$$

Since $\pi(F)$ is simply connected we conclude that $R_{n}=\pi\left(H_{n}^{*}\right)$ is of finite topological type. 


\section{Some examples}

In the following examples we assume that $\Gamma$ is a Fuchsian group without elliptic elements and that $\boldsymbol{D} / \Gamma$ is conformally equivalent to a domain $R \subset \hat{\boldsymbol{C}}=\boldsymbol{C} \cup\{\infty\}$. Let $f$ denote a universal covering map of $\boldsymbol{D}$ onto $R$.

Example 1 (compare [10]). Let

$$
R=D \backslash E, \quad \operatorname{cap} E=0 .
$$

In his example mentioned before, Patterson [8, p. 289] chooses $E=\{\lambda(0): \lambda \in A$, $\lambda \neq \imath\}$ where $\Lambda$ is a finitely generated Fuchsian group of the first kind. Let

$$
T=\left\{e^{i \theta}: \Delta_{\alpha}\left(e^{i \theta}, \delta\right) \subset R \text { for some } \alpha>0, \delta>0\right\} ;
$$

see (2.1). We shall show that

$$
\Gamma \text { of fully accessible type } \Leftrightarrow \text { mes } T=2 \pi \text {. }
$$

This motivates the term "fully accessible".

The Green's function of $R$ with respect to 0 is $\log 1 / \mid w$ because cap $E=0$. Hence the singular Green's lines [1], [15] are the radial segments $\left(\varrho e^{i \theta}, e^{i \theta}\right)$ where $\varrho e^{i \theta} \in E$. The starlike domain $R^{*}$ obtained by deleting these segments from $R$ is the Green's star domain. It is easy to see from (4.1) that

$$
T \backslash\left\{e^{i \theta}: \varrho e^{i \theta} \in E\right\}=\left\{e^{i \theta}: \Delta_{\alpha}\left(e^{i \theta}, \delta^{*}\right) \subset R^{*} \text { for some } \alpha>0, \delta^{*}>0\right\} .
$$

Since cap $E=0$ it follows that the set $T^{*}$ of points $e^{i \theta}$ where $R^{*}$ is tangential to $\partial \boldsymbol{D}$ satisfies mes $T^{*}=$ mes $T$. Hence (4.2) holds by [11, Corollary]; the set denoted by $g(G)$ in $\left[11\right.$, p. 163] is our $R^{*}$.

Example 2. Let $R$ be a domain in $\hat{\boldsymbol{C}}$ with

$$
\partial R=E_{0} \cup \bigcup_{k=1}^{\infty} J_{k}, \operatorname{cap} E_{0}=0
$$

where $J_{k}$ are disjoint open Jordan arcs with $J_{k} \cap \overline{\partial R \backslash J_{k}}=\emptyset$. Since the function $f$ omits an arc it is of bounded characteristic. Hence the angular limit $f(\zeta)$ exists for almost all $\zeta \in \partial \boldsymbol{D}$. If $f(\zeta) \in J_{k}$ for some $k$ then $f$ is continuous at $\zeta$, by Carathéodory's theorem on conformal mappings, and it follows that $\zeta$ does not belong to the limit set $L(\Gamma)$. Since cap $E_{0}=0$ the Privalov uniqueness theorem [14, p. 210] shows that

$$
\operatorname{mes}\left\{\zeta \in \partial D: f(\zeta) \in E_{0}\right\}=0 \text {. }
$$

Since $f(\zeta) \in \partial R$ we conclude from (4.3) that mes $L(\Gamma)=0$. Hence $\Gamma$ is of fully accessible type. 
Example 3. Let $E=C \backslash R$ lie on the rectifiable Jordan arc $C$ and let $l$ denote the arc length measure on $C$. We shall show that

$$
l(E)>0 \Rightarrow \Gamma \text { of accessible type. }
$$

The curve $C$ has a tangent for almost all $a \in E$. Since $C \backslash C \subset G$ we conclude that there is an open triangle of vertex $a$ that lies in $G$. If $l(E)>0$ it follows [13, Lemma 1] that $f$ has a finite angular derivative on a set on $\partial \boldsymbol{D}$ of positive measure. Hence $\Gamma$ is of accessible type by [12, Remark on p. 293].

Example 4. We prove now the converse of (4.4) for the special case that $E=C \backslash R \subset \boldsymbol{R}$. Let cap $E>0$ so that $\Gamma$ is of convergence type [6, p. 213]. We claim that

$$
l(E)>0 \Leftrightarrow \quad \Gamma \text { of accessible type. }
$$

Let $f(0)=\infty$. Since $f(F)$ is a simply connected subdomain of $R$ that is symmetric with respect to $\boldsymbol{R}$ we see that $f(F)=\hat{\boldsymbol{C}} \backslash I$ where $I$ is the smallest closed interval containing $E$.

Let $B=\partial F \cap \partial D$. Then $f(B) \subset E=\partial R$. Since $F$ is bounded by a rectifiable Jordan curve and since $f$ maps $F$ conformally onto $\hat{C} \backslash I$, it follows from $l(E)=0$ by the Riesz theorem [9, p. 320] that mes $B=0$. Hence $\Gamma$ is not of accessible type. This makes the term "accessible" appear somewhat incongruous.

\section{References}

[1] Brelot, M., and G. Choquet: Espaces et lignes de Green. - Ann. Inst. Fourier (Grenoble) 3, 1951, 199-263.

[2] Constantinescu, C., and A. Cornea: Ideale Ränder Riemannscher Flächen. - Ergebnisse der Mathematik und ihrer Grenzgebiete 32, Springer-Verlag, Berlin-GöttingenHeidelberg, 1963.

[3] Greenberg, L.: Finiteness theorems for Fuchsian and Kleinian groups. - Discrete groups and automorphic functions, Proceedings of an instructional conference organized by the London Mathematical Society and the University of Cambridge, Cambridge 1975, edited by W. J. Harvey, Academic Press, London-New York-San Francisco, 1977, 199-257.

[4] Herns, M.: On the principle of harmonic measure. - Comment Math. Helv. 33, 1959, 47-58.

[5] McMillan, J. E.: Boundary behavior of a conformal mapping. - Acta Math. 123, 1969, $43-67$.

[6] Nevanlinna, R.: Eindeutige analytische Funktionen, 2nd edition. - Springer-Verlag, BerlinGöttingen-Heidelberg, 1953.

[7] Patterson, S. J.: Spectral theory and Fuchsian groups. - Math. Proc. Cambridge Philos. Soc. $81,1977,59-75$.

[8] Patterson, S. J.: Some examples of Fuchsian groups. - Proc. London Math. Soc. (3) 39, 1979, 276-298.

[9] Pommerenke, Сh.: Univalent functions. - Vandenhoeck \& Ruprecht, Göttingen, 1975. 
[10] Pommerenke, Сн.: On the Green's function of Fuchsian groups. - Ann. Acad. Sci. Fenn. Ser. A I Math. 2, 1976, 409-427.

[11] Pommerenke, Сh.: On the Green's fundamental domain. - Math. Z. 156, 1977, 157-164.

[12] Pommerenke, СH. On the angular derivative and univalence. - Anal. Math. 3, 1977, 291 297.

[13] Pommerenke, Сh.: Polymorphic functions for groups of divergence type. - Math. Ann. $258,1982,353-366$.

[14] Priwalow, I. I.: Randeigenschaften analytischer Funktionen. - VEB Deutscher Verlag der Wissenschaften, Berlin, 1956.

[15] Sario, L., and M. NAKaI: Classification theory of Riemann surfaces. - Springer-Verlag, Berlin-Heidelberg-New York, 1970.

[16] Sullivan, D.: On the ergodic theory at infinity of an arbitrary discrete group of hyperbolic motions. - Riemann surfaces and related topics: Proceedings of the 1978 Stony Brook Conference, edited by Irwin Kra and Bernard Maskit, Annals of Mathematics Studies 97, Princeton University Press and University of Tokyo Press, Princeton, New Jersey, 1981, 465—496.

Technische Universität

Fachbereich Mathematik

D-1000 Berlin 12

Received 16 February 1982 